\title{
LINEAR COMBINATIONS OF ORTHOGONAL POLYNOMIALS GENERATING POSITIVE QUADRATURE FORMULAS
}

\author{
FRANZ PEHERSTORFER
}

\begin{abstract}
Let $p_{k}(x)=x^{k}+\cdots, k \in \mathbf{N}_{0}$, be the polynomials orthogonal on $[-1,+1]$ with respect to the positive measure $d \sigma$. We give sufficient conditions on the real numbers $\mu_{j}, j=0, \ldots, m$, such that the linear combination of orthogonal polynomials $\sum_{j=0}^{m} \mu_{j} p_{n-j}$ has $n$ simple zeros in $(-1,+1)$ and that the interpolatory quadrature formula whose nodes are the zeros of $\sum_{j=0}^{m} \mu_{j} p_{n-j}$ has positive weights.
\end{abstract}

\section{INTRODUCTION}

Let $\sigma$ be a positive measure on $[-1,1]$ such that the support of $d \sigma$ contains an infinite set of points. In this paper we consider interpolatory quadrature formulas with positive weights, i.e., quadrature formulas of the form

$$
\int_{-1}^{+1} f(x) d \sigma(x)=\sum_{j=1}^{n} c_{j} f\left(x_{j}\right)+R_{n}(f),
$$

where $-1<x_{1}<x_{2}<\cdots<x_{n}<1, c_{j}>0$ for $j=1, \ldots, n$, and $R_{n}(f)=0$ for $f \in \mathbf{P}_{2 n-1-m}, 0 \leq m \leq n \quad\left(\mathbf{P}_{n}\right.$ denotes as usual the set of polynomials of degree at most $n$ ). As in [6], such a quadrature formula is called a positive $(2 n-1-m, n, d \sigma)$ quadrature formula. If $\sigma$ is absolutely continuous on $[-1,1]$, with $\sigma^{\prime}(x)=w(x)$, we write also $(2 n-1-m, n, w)$ instead of $(2 n-1-m, n, d \sigma)$. Furthermore, we say that a polynomial $t_{n} \in \mathbf{P}_{n}$ generates a positive $(2 n-1-m, n, d \sigma)$ quadrature formula if $t_{n}$ has $n$ simple zeros $x_{1}<x_{2}<\cdots<x_{n}$ in $(-1,+1)$ and the interpolatory quadrature formula based on the nodes $x_{j}$ is a positive $(2 n-1-m, n, d \sigma)$ quadrature formula. Since the degree of exactness is $2 n-1-m$, we get with the help of (1.1) the well-known fact that such a polynomial $t_{n}$ is orthogonal to $\mathbf{P}_{n-1-m}$ with respect to $d \sigma$, and hence is of the form

$$
t_{n}(x)=\sum_{j=0}^{m} \mu_{j} p_{n-j}(x)
$$

Received January 11, 1989.

1980 Mathematics Subject Classification (1985 Revision). Primary 65D30; Secondary 33A65.

Key words and phrases. Quadrature formula, positive weights, orthogonal polynomials, zeros. 
where $\mu_{j} \in \mathbf{R}$ and $p_{k}(x)=x^{k}+\cdots, k \in \mathbf{N}_{0}$, denotes the polynomial of degree $k$ orthogonal with respect to $d \sigma$. For that reason we are interested in conditions on the numbers $\mu_{j}$ such that $t_{n}$ generates a positive $(2 n-1-m, n, d \sigma)$ quadrature formula. For small $m, m=1,2,3$, necessary and sufficient conditions on the numbers $\mu_{j}$ can be obtained from the general characterizations of positive quadrature formulas given by the author in [7, 8] (see in particu$\operatorname{lar}[8$, Theorem 2(b)]), by Sottas and Wanner [10] (note that the conditions given there do not imply that the nodes are in $(-1,+1)$ ), and recently by H. J. Schmid [9]. But for larger $m$ the computational work increases rapidly, and the conditions become very complex (see the examples given in $[9,10]$ ). Thus, the problem arises to find "simple and applicable" sufficient conditions on the numbers $\mu_{j}$ such that $\sum_{j=0}^{m} \mu_{j} p_{n-j}$ generates a positive $(2 n-1-m, n, d \sigma)$ quadrature formula. This problem is studied and partly solved in this paper by giving first a general sufficient condition on the $\mu_{j}$ 's, from which simpler conditions are derived.

\section{Preliminary Results}

In order to state our results, we need some known facts on polynomials orthogonal on $[-1,1]$, resp. orthogonal on the circumference of the unit circle $|z|=1$. Let us recall that the polynomials $p_{n}=x^{n}+\cdots, n \in \mathbf{N}$, orthogonal with respect to $d \sigma$ on $[-1,+1]$ satisfy a recurrence relation of the form

$$
p_{n}(x)=\left(x-\alpha_{n}\right) p_{n-1}(x)-\lambda_{n} p_{n-2}(x) \text { for } n \in \mathbf{N},
$$

where $p_{-1}=0, p_{0}=1, \alpha_{n} \in(-1,+1)$ for $n \in \mathbf{N}$, and $\lambda_{n}>0$ for $n \geq 2$. $p_{n}^{(1)}, n \in \mathbf{N}_{0}$, denotes the so-called associated polynomial, defined by

$$
p_{n}^{(1)}(x)=\frac{1}{d_{0}} \int_{-1}^{+1} \frac{p_{n+1}(x)-p_{n+1}(t)}{x-t} d \sigma(t),
$$

where $d_{0}=\int_{-1}^{+1} d \sigma(t)$. Note that the $p_{n}^{(1)}$ 's are polynomials of degree $n$ with leading coefficient one, which satisfy the following recurrence relation (see e.g. [2, Chapter 3, §4])

$$
p_{n}^{(1)}(x)=\left(x-\alpha_{n+1}\right) p_{n-1}^{(1)}(x)-\lambda_{n+1} p_{n-2}^{(1)}(x) \text { for } n \in \mathbf{N},
$$

where the $\alpha_{n}$ 's and $\lambda_{n}$ 's are determined by (2.1).

We are now ready to state the first simple characterization of positive quadrature formulas.

Lemma 1. Let $n, m \in \mathbf{N}_{0}, n \geq m$, and let $\mu_{j} \in \mathbf{R}$ for $j=0, \ldots, m, \mu_{0} \neq 0$. Then $\sum_{j=0}^{m} \mu_{j} p_{n-j}$ generates a positive $(2 n-1-m, n, d \sigma)$ quadrature formula if and only if $\sum_{j=0}^{m} \mu_{j} p_{n-j}$ has $n$ simple zeros in $(-1,+1)$ and the zeros of $\sum_{j=0}^{m} \mu_{j} p_{n-j}$ and $\sum_{j=0}^{m} \mu_{j} p_{n-1-j}^{(1)}$ separate each other.

Proof. Setting

$$
t_{n}=\sum_{j=0}^{m} \mu_{j} p_{n-j} \quad \text { and } \quad t_{n-1}^{(1)}=\sum_{j=0}^{m} \mu_{j} p_{n-1-j}^{(1)},
$$


we get for the weights $c_{j}$, using relation (2.2),

$$
c_{j}=\int_{-1}^{+1} \frac{t_{n}(x)}{\left(x-x_{j}\right) t_{n}^{\prime}\left(x_{j}\right)} d \sigma(x)=d_{0} \frac{t_{n-1}^{(1)}\left(x_{j}\right)}{t_{n}^{\prime}\left(x_{j}\right)} \text { for } j=1, \ldots, n .
$$

Hence the conditions $c_{j}>0$ for $j=1, \ldots, n$ are equivalent to the interlacing property of the zeros of $t_{n}$ and $t_{n-1}^{(1)}$.

Next, denote by $P_{n}(z)=z^{n}+\cdots, n \in \mathbf{N}_{0}$, the polynomial orthogonal on $[0,2 \pi]$ with respect to the positive measure

$$
\psi(\phi)= \begin{cases}-\sigma(\cos \phi) & \text { for } \phi \in[0, \pi] \\ \sigma(\cos \phi) & \text { for } \phi \in(\pi, 2 \pi]\end{cases}
$$

i.e.,

$$
\int_{0}^{2 \pi} e^{-i k \phi} P_{n}\left(e^{i \phi}\right) d \psi(\phi)=0 \text { for } k=0, \ldots, n-1 .
$$

Note if $\sigma$ is absolutely continuous on $[-1,+1]$ and $\sigma^{\prime}(x)=w(x)$, then $\psi$ is absolutely continuous with $\psi^{\prime}(\phi)=w(\cos \phi)|\sin \phi|$ for $\phi \in[0,2 \pi]$. It is well known (polynomials orthogonal on the unit circle are studied extensively in [3]) that the $P_{n}$ 's satisfy a recurrence relation of the type

$$
P_{n}(z)=z P_{n-1}(z)-a_{n-1} P_{n-1}^{*}(z) \text { for } n \in \mathbf{N},
$$

where $a_{n} \in(-1,+1)$ for $n \in \mathbf{N}_{0}$, and where $P_{n}^{*}(z)=z^{n} P_{n}\left(z^{-1}\right)$ denotes the reciprocal polynomial of $P_{n}$. The reason that the parameters $a_{n}$ are real and have absolute value less than one consists in the facts that $\psi$ is odd with respect to $\pi$ and that $\psi$ has an infinite set of points of increase (see [3, p. 5]). Furthermore, let $\Omega_{n}(z)=z^{n}+\cdots$ be defined by the recurrence relation

$$
\Omega_{n}(z)=z \Omega_{n-1}(z)+a_{n-1} \Omega_{n-1}^{*}(z) \text { for } n \in \mathbf{N} .
$$

$\Omega_{n}$ is called the associated polynomial of $P_{n}$. It is well known that both polynomials $P_{n}$ and $\Omega_{n}, n \geq 1$, have all their zeros in the open unit disk $|z|<1$. The following relations hold between polynomials $p_{n}$ orthogonal on $[-1,1]$ with respect to $d \sigma$ and polynomials $P_{n}$ :

$$
\begin{gathered}
p_{n}(x)=2^{-n+1} \operatorname{Re}\left\{z^{-n+1} P_{2 n-1}(z)\right\}, \\
p_{n-1}^{(1)}(x)=2^{-n+1} \operatorname{Im}\left\{z^{-n+1} \Omega_{2 n-1}(z)\right\} / \sin \phi,
\end{gathered}
$$

where $x=\frac{1}{2}\left(z+z^{-1}\right), z=e^{i \phi}, \phi \in[0, \pi]$. The parameters $\left(a_{n}\right)$ are given by $[3,(31.4)]$

$$
a_{2 n-1}=1-\left(u_{n}+v_{n}\right) \text { and } a_{2 n}=\frac{v_{n}-u_{n}}{v_{n}+u_{n}}
$$

where

$$
u_{n}=\frac{p_{n+1}(1)}{p_{n}(1)} \quad \text { and } \quad v_{n}=-\frac{p_{n+1}(-1)}{p_{n}(-1)}
$$


Moreover,

$$
a_{2 n}=0 \text { for } n \in \mathbf{N}_{0} \text {, if } \sigma(x)=-\sigma(-x) \text { a.e. on }[-1,1] \text {. }
$$

For example, we obtain for the Jacobi polynomials $p_{n}^{(\alpha, \beta)}(x)=x^{n}+\cdots$ which are orthogonal on $[-1,1]$ with respect to the weight function $w^{(\alpha, \beta)}(x)=$ $(1-x)^{\alpha}(1+x)^{\beta}, \alpha, \beta>-1$, that the corresponding parameters $a_{n}^{(\alpha, \beta)}$ appearing in the recurrence relation of $P_{n}^{(\alpha, \beta)}(z)=z^{n}+\cdots$ are given by

$$
a_{2 n+1}^{(\alpha, \beta)}=-\frac{\alpha+\beta+1}{\alpha+\beta+2 n+3}, \quad a_{2 n}^{(\alpha, \beta)}=\frac{\beta-\alpha}{\alpha+\beta+n+2} \quad \text { for } n \in \mathbf{N}_{0} .
$$

Hence we get for the ultraspherical case $p_{n}^{(\lambda)}(x):=p_{n}^{(\lambda-1 / 2, \lambda-1 / 2)}(x)$ and $w^{(\lambda)}(x)$ $=\left(1-x^{2}\right)^{\lambda-1 / 2}$ that

$$
a_{2 n+1}^{(\lambda)}=-\frac{\lambda}{n+1+\lambda} \quad \text { and } \quad a_{2 n}^{(\lambda)}=0 \text { for } n \in \mathbf{N}_{0},
$$

and in particular for the Chebyshev case, i.e., for the case where $\lambda=0$ and $w(x)=\left(1-x^{2}\right)^{-1 / 2}$, that

$$
a_{n}=0 \quad \text { for } n \in \mathbf{N}_{0}, \quad \Omega_{n}(z)=P_{n}(z)=z^{n} \quad \text { for } n \in \mathbf{N}_{0} .
$$

Finally, we shall need

Lemma 2. Let $n \in \mathbf{N}$ and $l \in \mathbf{Z}$ with $2|l| \leq n$. Assume that the real polynomial $t_{n}(z)=z^{n}+\cdots$ has all its zeros in the open unit disk $|z|<1$. Then the cosinepolynomial $\operatorname{Re}\left\{z^{-l} t_{n}(z)\right\}$, resp. the sine-polynomial $\operatorname{Im}\left\{z^{-l} t_{n}(z)\right\}, z=e^{i \phi}$, $\phi \in[0, \pi]$, has $n-l$ zeros $\phi_{j}$ in $(0, \pi)$, resp. $n-l-1$ zeros $\psi_{j}$ in $(0, \pi)$, and their zeros separate each other, i.e, $0<\phi_{1}<\psi_{1}<\phi_{2}<\cdots<\psi_{n-l-1}<$ $\phi_{n-l}<\pi$.

Proof. Since $\operatorname{Re}\left\{z^{-l} t_{n}(z)\right\}$ (respectively $\operatorname{Im}\left\{z^{-l} t_{n}(z)\right\}$ ) is zero at $z=e^{i \phi}$, $\phi \in(0,2 \pi)$, if and only if

which is equivalent to

$$
z^{-2 l} \frac{t_{n}(z)}{\overline{t_{n}(z)}}=-1 \quad(\text { respectively }+1)
$$

$$
\arg z^{n-2 l}+\arg \frac{t_{n}(z)}{t_{n}^{*}(z)}=(2 k-1) \pi \quad(\text { respectively } 2 k \pi),
$$

$k \in \mathbf{N}_{0}$, we get, taking into consideration the fact that $\arg t_{n}\left(e^{i \phi}\right) / t_{n}^{*}\left(e^{i \phi}\right)$ increases from 0 to $2 n \pi$ if $\phi$ varies from 0 to $2 \pi$, that both $\operatorname{Re}\left\{z^{-l} t_{n}(z)\right\}$ and $\operatorname{Im}\left\{z^{-l} t_{n}(z)\right\}$ have $2(n-l)$ zeros in $[0,2 \pi)$ and that their zeros separate each other. Observing that $\operatorname{Im}\left\{z^{-l} t_{n}(z)\right\}$ has a zero at $\phi=0$ and $\phi=\pi$, the assertion follows by the symmetry of trigonometric polynomials.

\section{MAIN RESUltS}

First, let us introduce the following polynomials, which play a crucial role in this paper. 
Definition. For given $n \in \mathbf{N}$ let the polynomials $Q_{\nu, 2 n-1}(z)=z^{\nu}+\cdots, \nu \in$ $\{0, \ldots, 2 n-1\}$, be defined by the recurrence relation

$$
\begin{aligned}
& Q_{\nu, 2 n-1}(z)=z Q_{\nu-1,2 n-1}(z)-a_{2 n-1-\nu} Q_{\nu-1,2 n-1}^{*} \\
& \text { for } \nu=1, \ldots, 2 n-1,
\end{aligned}
$$

where $Q_{0,2 n-1}=1$ and the $a_{2 n-1-\nu}$ 's are the parameters appearing in the recurrence relation $(2.5)$ of the $P_{n}$ 's.

The polynomials $Q_{\nu, 2 n-1}$ have the following important properties.

Lemma 3. Let $n \in \mathbf{N}$. The following propositions hold:

(a) $\prod_{\kappa=0}^{\nu-1}\left(1-\left|a_{2 n-2-\kappa}\right|\right) \leq\left|Q_{\nu, 2 n-1}^{*}(z)\right| \leq \prod_{\kappa=0}^{\nu-1}\left(1+\left|a_{2 n-2-\kappa}\right|\right)$ for $|z| \leq 1$, where $\nu \in\{0, \ldots, 2 n-1\}$. Moreover, $Q_{\nu, 2 n-1}$ has all zeros in $|z|<1$.

(b) Let $\nu \in\{0, \ldots, n-1\}$; then $\left(z=e^{i \phi}, x=\cos \phi, \phi \in[0, \pi]\right)$

$$
p_{n}(x)=2^{-n+1} \operatorname{Re}\left\{z^{-n+1} Q_{2 \nu, 2 n-1}(z) P_{2(n-\nu)-1}(z)\right\}
$$

and

$$
p_{n-1}^{(1)}(x)=2^{-n+1} \operatorname{Im}\left\{z^{-n+1} Q_{2 \nu, 2 n-1}(z) \Omega_{2(n-\nu)-1}(z)\right\} / \sin \phi .
$$

Proof. (a) follows immediately from (3.1) and [3, (26.6)].

(b) We first note that the recurrence relations (2.5), resp. (3.1), imply (see $[3,(3.6)])$ that

$$
P_{n}^{*}(z)=P_{n-1}^{*}(z)-a_{n-1} z P_{n-1}(z) \text { for } n \in \mathbf{N},
$$

and

$$
\begin{aligned}
Q_{\nu, 2 n-1}^{*}(z)=Q_{\nu-1,2 n-1}^{*}(z)-a_{2 n-1-\nu} & z Q_{\nu-1,2 n-1}(z) \\
& \text { for } \nu=1, \ldots, 2 n-1 .
\end{aligned}
$$

With the help of all these recurrence relations it follows by induction arguments that

$$
z P_{2 n-1}(z)+P_{2 n-1}^{*}(z)=z Q_{\nu, 2 n-1}(z) P_{2 n-1-\nu}(z)+Q_{\nu, 2 n-1}^{*}(z) P_{2 n-1-\nu}^{*}(z),
$$

which, in view of $(2.7)$ and taking into consideration the fact that for $z=e^{i \phi}$

$$
2 \operatorname{Re}\left\{z^{-n+1} P_{2 n-1}(z)\right\}=z^{-n}\left(z P_{2 n-1}(z)+P_{2 n-1}^{*}(z)\right),
$$

gives the first relation.

Analogously as above, one demonstrates that

$$
z \Omega_{2 n-1}(z)-\Omega_{2 n-1}^{*}(z)=z Q_{\nu, 2 n-1}(z) \Omega_{2 n-1-\nu}(z)-Q_{\nu^{\prime}, 2 n-1}^{*}(z) \Omega_{2 n-1-1}^{*}(z),
$$

which in conjunction with $(2.8)$ gives the second relation. 
The main result is now the following

Theorem 1. Let $n, m \in \mathbf{N}_{0}, m \leq n, \mu_{0}, \ldots, \mu_{m} \in \mathbf{R}$ and $\mu_{0} \neq 0$. Then $\sum_{j=0}^{m} \mu_{j} p_{n-j}$ generates a positive $(2 n-1-m, n, d \sigma)$ quadrature formula if $\sum_{j=0}^{m} \tilde{\mu}_{j} z^{j} Q_{2 m-2 j, 2(n-j)-1}(z)$, where $\tilde{\mu}_{j}=2^{j} \mu_{j}$, has all its zeros in the open unit disk $|z|<1$.

Proof. Putting

$$
t_{n}(x)=\sum_{j=0}^{m} \mu_{j} p_{n-j}(x) \quad \text { and } \quad t_{n-1}^{(1)}(x)=\sum_{j=0}^{m} \mu_{j} p_{n-1-j}^{(1)}(x),
$$

we get with the help of Lemma $3(\mathrm{~b})$ that $\left(z=e^{i \phi}, x=\cos \phi, \phi \in[0, \pi]\right)$

$$
t_{n}(x)=2^{-n+1} \operatorname{Re}\left\{z^{-m} q_{2 m}(z) z^{-(n-m)+1} P_{2(n-m)-1}(z)\right\}
$$

and

$$
t_{n-1}^{(1)}(x)=2^{-n+1} \operatorname{Im}\left\{z^{-m} q_{2 m}(z) z^{-(n-m)+1} \Omega_{2(n-m)-1}(z)\right\} / \sin \phi,
$$

where

$$
q_{2 m}(z)=\sum_{j=0}^{m} \tilde{\mu}_{j} z^{j} Q_{2 m-2 j, 2(n-j)-1}(z) .
$$

Assume now that $q_{2 m}$ has all its zeros in $|z|<1$. Since the same is true for $P_{2(n-m)-1}$, it follows from Lemma 2 that $t_{n}$ has $n$ simple zeros in $(-1,+1)$. Thus, by Lemma 1, it remains to demonstrate that the zeros of $t_{n}$ and $t_{n-1}^{(1)}$ separate each other.

Using the relation

$$
\operatorname{Re} a \operatorname{Re} b+\operatorname{Im} a \operatorname{Im} b=\operatorname{Re}\{a \bar{b}\},
$$

where $a, b \in \mathbf{C}$, we get for $z=e^{i \phi}$

$$
\begin{aligned}
\operatorname{Re} & \left\{z^{-(n-1)} q_{2 m}(z) P_{2(n-m)-1}(z)\right\} \operatorname{Re}\left\{z^{-(n-1)} q_{2 m}(z) \Omega_{2(n-m)-1}(z)\right\} \\
& +\operatorname{Im}\left\{z^{-(n-1)} q_{2 m}(z) P_{2(n-m)-1}(z)\right\} \operatorname{Im}\left\{z^{-(n-1)} q_{2 m}(z) \Omega_{2(n-m)-1}(z)\right\} \\
= & \left|q_{2 m}(z)\right|^{2} \operatorname{Re}\left\{P_{2(n-m)-1}(z) \overline{\Omega_{2(n-m)-1}(z)}\right\} \\
= & c\left|q_{2 m}(z)\right|^{2}, \quad c \in \mathbf{R}^{+},
\end{aligned}
$$

where the last equality follows from the known relation $[3,(5.6)]$

$$
\begin{aligned}
& P_{2(n-m)-1}(z) \Omega_{2(n-m)-1}^{*}(z)+\Omega_{2(n-m)-1}(z) P_{2(n-m)-1}^{*}(z) \\
& =\tilde{c} z^{2 n-2 m-1}, \quad \text { where } \tilde{c} \in \mathbf{R}^{+} .
\end{aligned}
$$

Considering relation (3.4) at the zeros $x_{j},-1<x_{1}<x_{2}<\cdots<x_{n}<1$, of $t_{n}(x)$ and taking into account that by Lemma 2 the zeros of $t_{n}(x)$ and $r_{n-1}(x):=\operatorname{Im}\left\{z^{-(n-1)} q_{2 m}(z) P_{2(n-m)-1}(z)\right\} / \sin \phi, x=\frac{1}{2}(z+1 / z), z=e^{i \phi}$, 
$\phi \in[0, \pi]$, separate each other, we obtain

$$
(-1)^{n-j} t_{n-1}^{(1)}\left(x_{j}\right)>0 \text { for } j=1, \ldots, n,
$$

which proves the interlacing property of $t_{n}$ and $t_{n-1}^{(1)}$ and thus the theorem.

Remark 1. From the general characterization of positive quadrature formulas given by the author in [7, Theorem 2] it follows with the help of relation (3.2) that the sufficient condition of Theorem 1 is also necessary if $2 m \leq n$.

From Theorem 1 we obtain, using some ideas of Cauchy and Kojima on the location of the zeros of polynomials (see [4, $\S 30$, in particular Exercise 6]), the following sufficient conditions which are easy to verify.

Corollary 1. Let $n, m \in \mathbf{N}_{0}, m \leq n, \mu_{0}, \ldots, \mu_{m} \in \mathbf{R}$ and $\mu_{0} \neq 0$. Put $A_{0}=\left|\mu_{0}\right|$,

$$
A_{j}=2^{j}\left|\mu_{j}\right| \frac{\prod_{\kappa=0}^{2 m-1-2 j}\left(1+\left|a_{2(n-j-1)-\kappa}\right|\right)}{\prod_{\kappa=0}^{2 m-1}\left(1-\left|a_{2(n-1)-\kappa}\right|\right)} \text { for } j=1, \ldots, m,
$$

and let $j_{\nu} \in\{0,1, \ldots, m\}, j_{0}:=0<j_{1}<\cdots<j_{m^{*}}$ be those indices for which $A_{j_{\nu}} \neq 0$ for $\nu=1, \ldots, m^{*}$ and $A_{j}=0$ for $j \in\{1, \ldots, m\} \backslash\left\{j_{0}, j_{1}, \ldots, j_{m^{*}}\right\}$. Then each of the following two conditions is sufficient that $\sum_{j=0}^{m} \mu_{j} p_{n-j}$ generates a positive $(2 n-1-m, n, d \sigma)$ quadrature formula:

(1) $\sum_{\nu=1}^{m^{*}} A_{j_{\nu}}<A_{0}$.

(2) $A_{j_{\nu}} \geq 2 A_{j_{\nu+1}}$ for $\nu=0, \ldots, m^{*}-2$ and $A_{j_{m^{*}-1}}>A_{j_{m^{*}}}$.

Proof. First let us note that condition (2) implies condition (1). In fact, applying successively the inequalities given in (2), we obtain

$$
A_{j_{0}} \geq A_{j_{1}}+A_{j_{1}} \geq A_{j_{1}}+A_{j_{2}}+A_{j_{2}} \geq \cdots>\sum_{\nu=1}^{m^{*}-1} A_{j_{\nu}}+A_{j_{m^{*}}}
$$

which is condition (1).

Next we show that condition (1) implies that

$$
q_{2 m}^{*}(z):=\sum_{j=0}^{m} \tilde{\mu}_{j} z^{j} Q_{2 m-2 j, 2(n-j)-1}^{*}(z), \quad \tilde{\mu}_{j}=2^{j} \mu_{j},
$$

has all zeros in $|z|>1$, which is equivalent to the fact that

$$
\sum_{j=0}^{m} \tilde{\mu}_{j} z^{j} Q_{2 m-2 j .2(n-j)-1}(z)
$$

has all zeros in $|z|<1$ and proves the corollary. Assume, to the contrary, that $q_{2 m}^{*}$ has a zero $\zeta$ in $|z| \leq 1$. Then it follows, using from Lemma 3 the fact that $Q_{2 m, 2 n-1}^{*}$ has no zero in $|z| \leq 1$, that

$$
\left|\mu_{0}\right|=\left|\sum_{j=1}^{m} \tilde{\mu}_{j} \zeta^{j} \frac{Q_{2 m-2 j, 2(n-j)-1}^{*}(\zeta)}{Q_{2 m, 2 n-1}^{*}(\zeta)}\right| \leq \sum_{j=1}^{m} A_{j}|\zeta|^{j} \leq \sum_{j=1}^{m} A_{j},
$$


where the first inequality follows with the help of Lemma 3, which is a contradiction to (1).

Let us give an illustrative

Example. Let $n, m \in \mathbf{N}_{0}, n>m$, and suppose that the parameters $a_{\nu}$ satisfy

$$
0<1 / \gamma \leq 1-\left|a_{\nu}\right| \text { for } \nu=2(n-m)-1, \ldots, 2 n-2 \text {. }
$$

Then we get by Corollary 1 that

$$
p_{n}-\mu_{m} p_{n-m}, \quad\left|\mu_{m}\right|<\left(2 \gamma^{2}\right)^{-m},
$$

generates a positive $(2 n-1-m, n, d \sigma)$ quadrature formula, where because of (2.10) the condition on $\left|\mu_{m}\right|$ can be replaced by $\left|\mu_{m}\right|<(2 \gamma)^{-m}$ if $\sigma$ is odd. In particular, we obtain for the Jacobi weight by a rough estimate of the parameters $a_{n}^{(\alpha, \beta)}$ from $(2.11)$ that

$$
p_{n}^{(\alpha, \beta)}-\mu_{m} p_{n-m}^{(\alpha, \beta)}, \quad\left|\mu_{m}\right|<2^{-3 m},
$$

generates a positive $\left(2 n-1-m, n,(1-x)^{\alpha}(1+x)^{\beta}\right)$ quadrature formula for each $n \geq m+\max \{2, \alpha+\beta+1+2|\beta-\alpha|\}$. In the ultraspherical case $\alpha=\beta=\lambda-1 / 2, \lambda \in(-1 / 2, \infty)$, the conditions on $\left|\mu_{m}\right|$, resp. $n$, can be replaced by $\left|\mu_{m}\right|<2^{-2 m}$ and $n \geq m+\max \{\lambda,-3 \lambda\}$.

Let us note in this connection that the conditions of Corollary 1 are in general too rough to get the known results (see [1]) on the positivity of $(n-1, n$, $\left.(1-x)^{\alpha}(1+x)^{\beta}\right)$ quadrature formulas generated by $p_{n}^{(a, b)}, a, b>-1$. But this is not surprising because the proof of such results requires very special properties of Jacobi polynomials.

In order to weaken the sufficient conditions of Corollary 1 , a better estimate for $\max _{0 \leq \phi \leq 2 \pi}\left|Q_{2 m-2 j, 2(n-j)-1}^{*}\left(e^{i \phi}\right) / Q_{2 m, 2 n-1}^{*}\left(e^{i \phi}\right)\right|$ than that one used in (3.6) would be needed.

In the following, let $T_{n}$, resp. $U_{n}$, denote the Chebyshev polynomial of the first, resp. second, kind of degree $n$ and $\widehat{T}_{n}(x)=2^{-n+1} T_{n}(x)=x^{n}+\cdots$, resp. $\widehat{U}_{n}(x)=2^{-n} U_{n}(x)=x^{n}+\cdots$. For the case of the Chebyshev distribution $d \sigma(x)=\left(1-x^{2}\right)^{-1 / 2} d x$ we get in view of (2.13) particularly simple conditions, which hold also for the distribution $d \sigma(x)=\left(1-x^{2}\right)^{1 / 2} d x$.

Corollary 2. Let $n, m \in \mathbf{N}_{0}, m \leq n, \mu_{0}, \ldots, \mu_{m} \in \mathbf{R}, \mu_{0} \neq 0$, and put $\tilde{\mu}_{j}=2^{j} \mu_{j}$ for $j=0, \ldots, m$. Then the following propositions hold:

(a) $\sum_{j=0}^{m} \mu_{j} \widehat{T}_{n-j}$ generates a positive $\left(2 n-1-m, n,\left(1-x^{2}\right)^{-1 / 2}\right)$ quadrature formula if $\sum_{j=0}^{m} \tilde{\mu}_{j} z^{m-j}$ has all its zeros in the open unit disk $|z|<1$. In particular (besides conditions (1) and (2) of Corollary 1), the condition

(3) $\tilde{\mu}_{0}>\tilde{\mu}_{1}>\cdots>\tilde{\mu}_{m}>0$

is sufficient that $\sum_{j=0}^{m} \mu_{j} \widehat{T}_{n-j}$ generates a positive $\left(2 n-1-m, n,\left(1-x^{2}\right)^{-1 / 2}\right)$ quadrature formula. 
(b) The sufficient conditions given in (a) (including conditions (1) and (2) of Corollary 1 with $a_{n}=0$ for $n \in \mathbf{N}_{0}$ ) are also sufficient for $\sum_{j=0}^{m} \mu_{j} \widehat{U}_{n-j}$ to generate a positive $\left(2 n-1-m, n,\left(1-x^{2}\right)^{1 / 2}\right)$ quadrature formula.

Proof. (a) The first statement follows immediately from Theorem 1. Since by the Kakeya-Eneström Theorem (see, e.g., [4]) condition (3) implies that $\sum_{j=0}^{m} \tilde{\mu}_{j} z^{m-j}$ has all zeros in $|z|<1$, part (a) is proved.

(b) We shall demonstrate, independently from Theorem 1 , that $\sum_{j=0}^{m} \mu_{j} \widehat{U}_{n-j}$ generates a positive $\left(2 n-1-m, n,\left(1-x^{2}\right)^{1 / 2}\right)$ quadrature formula if $\sum_{j=0}^{m} \tilde{\mu}_{j}$. $z^{m-j}$ has all zeros in $|z|<1$, which also implies all other statements of (b). Setting

$$
r_{n}(z)=z^{n-m} \sum_{j=0}^{m} \tilde{\mu}_{j} z^{j}
$$

and

$$
2^{n} t_{n}(x)=\sum_{j=0}^{m} \tilde{\mu}_{j} U_{n-j}(x)=\operatorname{Im}\left\{z r_{n}(z)\right\} / \sin \phi,
$$

we obtain, since, as is well known, the associated polynomial of $\widehat{U}_{k}$ is $\widehat{U}_{k-1}$, $k \in \mathbf{N}_{0}$, that the associated polynomial $t_{n-1}^{(1)}$ of $t_{n}$ with respect to $\left(1-x^{2}\right)^{1 / 2}$ is of the form

$$
2^{n-1} t_{n-1}^{(1)}(x)=\sum_{j=0}^{m} \tilde{\mu}_{j} U_{n-1-j}(x)=\operatorname{Im}\left\{r_{n}(z)\right\} / \sin \phi .
$$

Observing that

$$
\operatorname{Re}\left\{r_{n}(z)\right\} \frac{\operatorname{Im}\left\{z r_{n}(z)\right\}}{\sin \phi}-\operatorname{Re}\left\{z r_{n}(z)\right\} \frac{\operatorname{Im}\left\{r_{n}(z)\right\}}{\sin \phi}=\left|r_{n}(z)\right|^{2},
$$

we deduce with the help of Lemma 2, by considering relation (3.7) at the $n$ zeros of $t_{n}$, that $t_{n}$ and $t_{n-1}^{(1)}$ have interlacing zeros. In view of Lemma 1 the assertion is proved.

The sufficiency of condition (3) for the Chebyshev weight $\left(1-x^{2}\right)^{-1 / 2}$ is due to C. A. Micchelli [5], who derived this result in order to demonstrate that the ultraspherical polynomials $p_{n}^{(\lambda)}, 0 \leq \lambda<1$, generate a positive $\left(n-1, n,\left(1-x^{2}\right)^{-1 / 2}\right)$ quadrature formula. Let us mention in this connection (for a different approach see [5]) that for $-1 / 2<\lambda \leq 0$ the positivity can be demonstrated with the help of condition (1), using the simple fact that $T_{k}(1)=1$ for $k \in \mathbf{N}_{0}$. Proceeding similarly as in the proof of Corollary 2(b), it could also be demonstrated that Corollary 2(b) holds for the more general weight $(1-x)^{\alpha}(1+x)^{\beta}, \alpha, \beta \in\{-1 / 2,1 / 2\}$, a result which has been given by the author in $[8$, Corollary 2$]$, using different methods. 
Using the fact that the sufficient condition of Theorem 1 is also necessary if $2 m \leq n$ (see Remark 1), we get

Corollary 3. Let $n, m \in \mathbf{N}_{0}, 2 m \leq n, \mu_{0}, \ldots, \mu_{m} \in \mathbf{R}$ and $\mu_{0}, \mu_{m} \in \mathbf{R} \backslash\{0\}$. For $k \in\{0, \ldots, m\}$ put $A_{k}^{(k)}=2^{k}\left|\mu_{k}\right|$ and

$$
A_{j}^{(k)}=2^{j}\left|\mu_{j}\right| \frac{\prod_{\kappa=0}^{2 m-1-2 j}\left(1+\left|a_{2(n-j-1)-\kappa}\right|\right)}{\prod_{\kappa=0}^{2 m-1-2 k}\left(1-\left|a_{2(n-k-1)-\kappa}\right|\right)} \quad \text { for } j=0, \ldots, m, j \neq k .
$$

If there is a $k \in\{1, \ldots, m\}$ such that $A_{k}^{(k)}>\sum_{j=0, j \neq k}^{m} A_{j}^{(k)}$, then $\sum_{j=0}^{m} \mu_{j}$. $p_{n-j}$ does not generate a positive $(2 n-1-m, n, d \sigma)$ quadrature formula.

Proof. In view of Remark 1 it is sufficient to demonstrate that

$$
q_{2 m}^{*}(z):=\sum_{j=0}^{m} \tilde{\mu}_{j} z^{j} Q_{2 m-2 j, 2(n-j)-1}^{*}(z), \quad \tilde{\mu}_{j}=2^{j} \mu_{j},
$$

has at least one zero in $|z|<1$. With the help of Lemma 3 we get on the circumference $|z|=1$

$$
\begin{aligned}
\left|\tilde{\mu}_{k} z^{k} Q_{2 m-2 k, 2(n-k)-1}^{*}(z)\right| & \geq\left|\tilde{\mu}_{k}\right| \prod_{\kappa=0}^{2 m-1-2 k}\left(1-\left|a_{2(n-k-1)-\kappa}\right|\right) \\
& >\sum_{\substack{j=0 \\
j \neq k}}^{m}\left|\tilde{\mu}_{j}\right| \prod_{\kappa=0}^{2 m-1-2 j}\left(1+\left|a_{2(n-j-1)-\kappa}\right|\right) \\
& \geq\left|\sum_{\substack{j=0 \\
j \neq k}}^{m} \tilde{\mu}_{j} z^{j} Q_{2 m-2 j, 2(n-j)-1}^{*}(z)\right| .
\end{aligned}
$$

Using the fact that $Q_{2 m-2 k, 2(n-k)-1}^{*}$ has no zero in $|z|<1$, this implies by Rouché's Theorem that $q_{2 m}^{*}$ has $k$ zeros in $|z|<1$, which proves the assertion.

If one is interested only in such linear combinations of orthogonal polynomials whose zeros are simple and are in $(-1,+1)$, conditions (1) and (2) can be weakened in the following way.

Theorem 2. Let $n, m \in \mathbf{N}_{0}, m \leq n, \mu_{0}, \ldots, \mu_{m} \in \mathbf{R}$ and $\mu_{0} \neq 0$. Put $\left|B_{0}\right|=\mu_{0}$,

$$
B_{j}=2^{j}\left|\mu_{j}\right| / \prod_{\kappa=2(n-j)-1}^{2 n-2}\left(1-\left|a_{\kappa}\right|\right) \text { for } j=1, \ldots, m,
$$

and let $j_{\nu} \in\{0,1, \ldots, m\}, j_{0}:=0<j_{1}<\cdots<j_{m}$. be those indices for which $B_{j_{v}} \neq 0$ for $\nu=1, \ldots, m^{*}$ and $B_{j}=0$ for $j \in\{1, \ldots, m\} \backslash\left\{j_{0}, j_{1}, \ldots, j_{m^{*}}\right\}$. Then each of the following two conditions is sufficient that $\sum_{j=0}^{m} \mu_{j} p_{n-j}$ has $n$ 
simple zeros in $(-1,1)$ :

(1') $\sum_{\nu=1}^{m^{*}} B_{j_{\nu}}<B_{0}$.

(2') $B_{j_{\nu}} \geq 2 B_{j_{\nu+1}}$ for $\nu=0, \ldots, m^{*}-1$ and $B_{j_{m^{*}-1}}>B_{j_{m^{*}}}$.

Proof. Since by (2.7)

$$
\sum_{j=0}^{m} \mu_{j} p_{n-j}(x)=2^{-n+1} \operatorname{Re}\left\{\sum_{j=0}^{m} \tilde{\mu}_{j} z^{j} P_{2(n-j)-1}(z)\right\},
$$

where $\tilde{\mu}_{j}=2^{j} \mu_{j}$, we deduce with the help of Lemma 2 that $\sum_{j=0}^{m} \mu_{j} p_{n-j}(x)$ has $n$ simple zeros in $(-1,+1)$ if $\sum_{j=0}^{m} \mu_{j} z^{j} P_{2(n-j)-1}^{*}$ has all zeros in $|z|>1$. Observing that by relation (26.5) of [3]

$$
\max _{|z| \leq 1}\left|\frac{P_{2(n-j)-1}^{*}(z)}{P_{2 n-1}^{*}(z)}\right| \leq \frac{1}{\prod_{\kappa=2(n-j)-1}^{2 n-2}\left(1-\left|a_{\kappa}\right|\right)} \quad \text { for } j=1, \ldots, m,
$$

the assertion can be proved in the same way as Corollary 1 .

\section{BIBLIOGRAPHY}

1. R. Askey, Positive quadrature methods and positive polynomial sums, Approximation Theory. V (C. K. Chui, L. L. Schumaker, and J. D. Ward, eds.), Academic Press, New York, 1986, pp. 1-30.

2. T. S. Chihara, An introduction to orthogonal polynomials, Gordon and Breach, New York, 1978.

3. Ja. L. Geronimus, Polynomials orthogonal on a circle and their applications, Zap. NaučnoIssled. Inst. Mat. Mekh. Kharkov. Mat. Obshch. 19 (1948), 35-120; English transl., Amer. Math. Soc. Transl. 3 (1962), 1-78.

4. M. Marden, Geometry of polynomials, Amer. Math. Soc., Providence, R.I., 1966.

5. C. A. Micchelli, Some positive Cotes numbers for the Chebyshev weight function, Aequationes Math. 21 (1980), 105-109.

6. C. A. Micchelli and T. J. Rivlin, Numerical integration rules near Gaussian quadrature, Israel J. Math. 16 (1973), 287-299.

7. F. Peherstorfer, Characterization of positive quadrature formulas, SIAM J. Math. Anal. 12 (1981), 935-942.

8. Characterizations of quadrature formulas. II. SIAM J. Math. Anal. 15 (1984), 10211030.

9. H. J. Schmid, A note on positive quadrature rules, Rocky Mountain J. Math. 19 (1989), 395-404.

10. G. Sottas and G. Wanner. The number of positive weights of a quadrature formula, BIT 22 (1982), 339-352. 